\title{
Parametric Instabilities During High Power Helicon Wave Injection on DIII-D
}

\author{
M. Porkolab ${ }^{1}$ and R.I. Pinsker ${ }^{2}$ \\ ${ }^{1}$ Massachusetts Institute of Technology, Plasma Science and Fusion Center, Cambridge, Massachusetts 02139, USA \\ ${ }^{2}$ General Atomics, P.O. Box 85608, San Diego, California 92186-5608, USA
}

\begin{abstract}
High power helicon (whistler) waves at a frequency of $0.47 \mathrm{GHz}$ are being considered for efficient off-axis current generation in high performance DIII-D plasmas and in K-Star [3]. The need for deploying helicon waves for current profile control has been noted in previous publications since penetration to the core of reactor grade plasmas is easier than with lower hybrid slow waves (LHCD) which suffer from accessibility limitations and strong electron Landau absorption in fusion grade high temperature plasmas. In this work we show that under typical experimental conditions in present day tokamaks with $1 \mathrm{MW}$ of RF power coupled per antenna, the associated perpendicular electric fields of the order of $40 \mathrm{kV} / \mathrm{m}$ can drive strong parametric decay instabilities near the lower hybrid layer. The EXB and polarization drift velocities which are the dominant driver of the PDI can be comparable to the speed of sound in the outer plasma layers, a key measure of driving PDI instabilities. Here we calculate growth rates and convective thresholds for PDIs, and we find that decay waves into hot ion lower hybrid waves and ion cyclotron quasi modes dominate in the vicinity of the lower hybrid layer, possibly leading to pump depletion. Such instabilities in future reactor grade high temperature plasmas are less likely.
\end{abstract}

\section{Introduction}

We consider the possibility of parametric decay instabilities (PDI) in the proposed $1 \mathrm{MW}$ helicon wave (whistler or fast wave at frequencies below the central lower hybrid frequency in a tokamak plasma) current drive experiments on DIII-D $[1,2]$. The importance of introducing such a current drive method was already recognized in the ARIES reactor studies where it was necessary to introduce the fast lower hybrid wave (helicon wave in current use) in the $1 \mathrm{GHz}$ range to provide some of the necessary driven current in the outer region of the plasma core, yet well inside the pedestal region [3]. Previous work postulated [4] that significant levels of PDI would be unlikely in such experiments due to the weaker RF electric fields of these predominantly electromagnetic waves, in contrast to those of the quasilongitudinal (electrostatic) slow lower hybrid wave $[5,6$, $7,8]$. In this work we show that this is not necessarily the case under present day experimental conditions in medium size tokamaks, e.g., DIII-D, where $1 \mathrm{MW}$ of RF power at $476 \mathrm{MHz}$ is proposed to be injected using a "comb-line" traveling wave antenna [2]. We note that similar experiments are also in the planning stage for the KSTAR tokamak [9]. Under such conditions perpendicular electric fields of $40 \mathrm{kV} / \mathrm{m}$ may be generated which then drive ExB velocities and also, comparable values of the polarization drift velocity. While these are weaker fields than those typical for the slow wave $\left(\mathrm{E}_{/ /}=300 \mathrm{kV} / \mathrm{m}\right.$ and the perpendicular value of $\mathrm{E}$ is even larger) they are comparable to the local acoustic speed in the relatively low density and temperature scrape-off layer near the local lower hybrid frequency and may be sufficient to drive PDI $[4,5]$.

In what follows we will summarize the directly relevant theoretical work of the author from past years and proceed to calculate growth rates for typical DIII-D parameters. We will then estimate convective growth rates of the expected decay into ion cyclotron quasimodes and the slow lower hybrid waves, in particular the hot ion plasma waves, under the greatly simplified mathematical formulation of a slab geometry $[5,6]$. We find that under the idealized conditions of our calculations, significant convective growth may occur near the lower hybrid resonance layer in the edge plasma. However, under reactor like conditions, where the electric fields may remain the same but the edge plasma parameters, in particular the temperatures will be much higher, such instabilities may not be important. However, this remains the subject of future studies.

\section{Parametric Instabilities in the Lower Hybrid Range of Frequencies}

We shall consider the theory of parametric decay instabilities (PDI) in the lower hybrid range of frequencies as in past works $[5,6]$, i.e., assume that the

\footnotetext{
Corresponding author: porkolab@psfc.mit.edu
} 
injected high amplitude RF wave (pump wave) has a wavelength that is much longer than that of the decay waves, and hence we shall calculate growth rates in the so-called "dipole" approximation, namely $\mathbf{k}_{\mathbf{0}}<<\mathbf{k}_{\mathbf{j}}$, where $\mathrm{j}=1,2$ corresponds to the decay or daughter waves, and in fact we shall set $\mathbf{k}_{\mathbf{0}}=\mathbf{0}$ (where $\mathbf{j}=1,2$ corresponds to the decay or daughter waves). The daughter waves in the present work will either be hot ion plasma waves at the lower sideband $\omega_{2}=\left(\omega_{0}-\omega\right)$, (an ensemble of ion Bernstein waves propagating through high cyclotron harmonic frequencies nearly perpendicular to the weakly inhomogeneous magnetic field), and ion cyclotron quasi-modes (heavily cyclotron damped electrostatic response of the plasma near the beat frequency, $\left(\omega_{0}-\omega_{2}\right)=n \Omega_{i}$, where $n$ is an integer and $\Omega_{\mathrm{i}}$ is the angular ion cyclotron frequency ) $[5,10]$. The selection rules in the dipole approximation reduce to $\mathbf{k}_{\mathbf{1}}=-\mathbf{k}_{\mathbf{2}}$ and this greatly simplifies the theory which then can be derived even for relatively large amplitude pump electric fields $[5,6,11]$. The growth rates for decay waves (of order $1 \mathrm{MHz}$ ) are much larger than the bandwith of the pump wave ( $1 \mathrm{kHz}$ range). The relevant theoretical results for PDI will be summarized by the equations in the sections below.

\subsection{Parametric Decay Instabilities in the Large Amplitude Limit}

We start with the Vlasov equation in the presence of the large amplitude pump wave,

$$
\begin{gathered}
E_{0}(x, t)=\left(E_{0 x} \hat{x}+E_{0 z} \hat{z}\right) \cos \omega_{0} t, \\
\partial f / \partial t+\mathbf{v} \cdot \nabla f+(q / m)\left(\frac{\mathbf{v} \times \mathbf{B}}{c}+\mathbf{E}+\right. \\
\left.\mathbf{E}_{0} \cos \omega_{0} t\right) \cdot \nabla_{v} f=0
\end{gathered}
$$

the fundamental parametric instability dispersion relationship in the dipole approximation is given by $[5$, 11]:

$$
\begin{aligned}
& 1+\frac{1}{\chi_{i}}=J_{0}^{2}(\mu) \frac{\chi_{e}}{1+\chi_{e}}+J_{1}^{2}(\mu)\left(\frac{\chi_{e}^{+}}{1+\chi_{e}^{+}}+\frac{\chi_{e}^{-}}{1+\chi_{e}^{-}}\right) \\
& +\frac{J_{0}^{2}(\mu) J_{1}^{2}(\mu)\left\{\left[\chi_{e} /\left(1+\chi_{e}\right)\right]-\left[\chi_{e}^{+} /\left(1+\chi_{e}^{+}\right)\right]\right\}^{2}}{1+\left(1 / \chi_{i}^{+}\right)-J_{0}^{2}(\mu)\left[\chi_{e}^{+} /\left(1+\chi_{e}^{+}\right)\right]-J_{1}^{2}(\mu)\left[\chi_{e} /\left(1+\chi_{e}\right.\right.} \\
& \frac{J_{0}^{2}(\mu) J_{1}^{2}(\mu)\left\{\left[\chi_{e} /\left(1+\chi_{e}\right)\right]-\left[\chi_{e}^{-} /\left(1+\chi_{e}^{-}\right)\right]\right]^{2}}{1+\left(1 / \chi_{i}^{-}\right)-J_{0}^{2}(\mu)\left[\chi_{e}^{-} /\left(1+\chi_{e}^{-}\right)\right]-J_{1}^{2}(\mu)\left[\chi_{e} /(1+\chi\right.}
\end{aligned}
$$

where

$$
\boldsymbol{\mu}=\left(e / m_{e}\right)\left[\left(\frac{E_{0 \|} k_{\| 1}}{\omega_{0}^{2}}+\frac{\mathbf{E}_{0 \perp} \cdot \mathbf{k}_{\perp}}{\omega_{0}^{2}-\omega_{c e}^{2}}\right)^{2}+\frac{\left|\mathbf{E}_{0 \perp} \times \mathbf{k}_{\perp}\right|^{2} \omega_{c e}^{2}}{\left(\omega_{0}^{2}-\omega_{c e}^{2}\right)^{2} \omega_{0}^{2}}\right]^{1 / 5}
$$

provides the coupling between the low frequency quasimodes and the lower hybrid ion plasma waves at the sidebands. The parallel and perpendicular components of the pump wave electric field are indicated by the appropriate subscripts, and the parallel and perpendicular components of the wave numbers of the daughter waves are also indicate appropriately. We recognize the three terms in $\mu$, as the parallel oscillating drift (or quiver) velocity, the perpendicular polarization drift velocity and the EXB drift velocity, respectively. The appropriate electrostatic susceptibility of electrons and ions is designated by the Greek alphabet symbol $\chi_{e}$ or $\chi_{i}$, respectively [5] and the lower $\left(\omega_{0}-\omega\right)$ or upper sideband $\left(\omega_{0}+\omega\right)$ responses are designated by the super $\left(^{-}\right)$or super $\left({ }^{+}\right)$symbols, respectively, while the susceptibilities at the low frequency $\omega=\mathrm{n} \Omega_{\mathrm{i}}$ (ion cyclotron quasi-modes in the present case) response have no superscripts. This dispersion has been configured into a numerical computer code MPPDI in the 1970s, including all orders of the electron and ion Larmor radius through retention of the modified Bessel functions in the hot electrostatic linear dispersion relationships to at least 50 cyclotron harmonics, and electron and ion Landau and cyclotron damping are also accounted for [5]. This code has been tested numerous times in the last 4 decades and is still being use at MIT by various students and research scientists, including the author. In the second half of the paper we shall present numerical results of the growth rates and convective thresholds of the most unstable modes corresponding to the planned helicon experiments in DIII-D.

\subsection{Parametric Decay into Two Weakly Damped Modes}

For small values of $\mu<<1$, the ordinary Bessel function $\mathrm{J}$ can be expanded in a power series and various terms combined into a compact form, equivalent to the usual PDI dispersion relationships. Indeed, it was shown in our earlier papers $[5,6,10]$ that the familiar PDI dispersion relationship for either resonant decay instability, the OTSI instability, and for decay into quasi modes (nonlinear Landau damping) can be readily deduced from Eq. (2) and are of the following form:

(a) Resonant decay into two weakly damped modes:

$$
\epsilon(\omega)+\frac{\mu^{2}}{4} \chi_{i}(\omega)\left[\chi_{e}(\omega)\right]\left[\frac{1}{\epsilon\left(\omega-\omega_{0}\right)}+\frac{1}{\epsilon\left(\omega+\omega_{0}\right)}\right]=0,
$$

with the threshold for temporal growth rates given by expansion about weakly damped normal modes,

$$
\left(\gamma+\gamma_{1}\right)\left(\gamma+\gamma_{2}\right)=\left.\left.\frac{\mu^{2}}{4} \chi_{i}\left(\omega_{1}\right) \chi_{e}\left(\omega_{1}\right) \frac{\partial \epsilon_{1}}{\partial \omega}\right|_{\omega_{1}} \frac{\partial \epsilon_{2}}{\partial \omega}\right|_{\omega_{2}}
$$

(b) Decay into low frequency quasi-modes:

$$
\begin{gathered}
\gamma=-\gamma_{2}+\mu^{2} \operatorname{Im}\left[\chi_{e}\left(\omega_{1}\right)\right]\left(\left|\chi_{i}\left(\omega_{1}\right)\right|^{2}+\operatorname{Re}\left[\chi_{i}\left(\omega_{1}\right)\right]\right) \\
\left.+\operatorname{Im}\left[\chi_{i}\left(\omega_{1}\right)\right]\left(\left|\chi_{e}\left(\omega_{1}\right)\right|^{2}+\operatorname{Re}\left[\chi_{e}\left(\omega_{1}\right)\right]\right) /|\partial \epsilon / \partial \omega| \omega_{2}\left|\epsilon\left(\omega_{1}\right)\right|^{2}\right\}
\end{gathered}
$$

where the quasi mode response is given by

$$
\left(\omega_{0}-\omega_{2}\right) / k_{\|} \simeq n \Omega / k_{\|} \simeq v_{t i}
$$


and the lower hybrid sideband wave in the straight hot ion orbit approximation (summation of high harmonic Bernstein waves) is given by :

$$
\omega^{2}=\omega_{L H}^{2}\left[1+\frac{k_{\|}^{2}}{k^{2}} \frac{m_{i}}{m_{e}}+\frac{3 k_{\perp}^{2} T_{i}}{\omega^{2} m_{i}}\left(1+\frac{1}{4} \frac{T_{e}}{T_{i}} \frac{\omega^{4}}{\omega_{c e}^{2} \omega_{c i}^{2}}\right)\right]
$$

Once we have the growth rates from either Eqs. 2 or 6 , we can obtain the convective threshold which has to be considered in all three dimensions, $x, y$ or $z[5,6,12]$. For example, in the $\mathrm{x}$ direction (radial direction in our case) the threshold is given by

$$
\frac{\gamma \Delta x}{\left|v_{2 x}\right|}=\frac{\gamma L_{x}}{\left|v_{2 x}\left(v_{2 z} / v_{2 x}-v_{0 z} / v_{0 x}\right)\right|}=\pi,
$$

or more generally we need to consider the diagram of convective losses in 3 dimension, as given in Ref. [12]. In fact, when we consider PDI near the lower hybrid frequency, we find that the minimum convective threshold is given when the perpendicular group velocity vanishes, and consequently convective losses in this direction vanish (ie, the second and third terms in Eq 8 balance), and therefore only convective losses in the $\mathrm{z}$ (toroidal) direction need to be considered. Additional stabilizing mechanisms in the radial direction include the density inhomogeneity, and in the poloidal direction the finite height of the antenna. Therefore in the relevant case the convective threshold is given by $\gamma \mathrm{L}_{\mathrm{z}} / \mathrm{V}_{\mathrm{gz}}=\pi$, where $\gamma$ is the growth rate obtained above from Eq 2 (or Eq 6) and $L_{z}$ is the effective antenna extent and $V_{g z}$ is the group velocity, both along the magnetic field, approximately in the $\mathrm{z}$ direction. Initial results for the helicon pump wave growth rates and convective losses are as follows. For 1.25 MW coupled power, GENRAY finds $45 \mathrm{kV} / \mathrm{m}$ or $450 \mathrm{Volt} / \mathrm{cm}$, see Ref.[1]. $E_{0 \mathrm{r}}$ is significantly smaller and $E_{0 z}$ is negligible. Near the lower hybrid mode conversion layer near the plasma edge (where $\mathrm{n}_{\mathrm{e}} \approx 3 \times 10^{18} \mathrm{~m}^{-3}, \mathrm{~T}_{\mathrm{e}}=30 \mathrm{eV}, \mathrm{T}_{\mathrm{e}}=\mathrm{T}_{\mathrm{i}}$, and $\mathrm{B}=1.3$ Tesla) based on results from extensive growth rate calculations from $\mathrm{Eq} \mathrm{2}$, we find maximum growth rates for $\mathrm{N}_{2 \mathrm{II}}=10.0, \mathrm{k}_{\mathrm{x}} \lambda_{\mathrm{D}}=0.08, \omega_{2} / \omega_{0}=0.077, \gamma / \omega_{0}=$ 0.0114 . Here for the pump wave we assumed $\mathrm{N}_{0 I I}=3.0$, and the appropriate selection rules for parallel wavenumbers were introduced into Eq.2 to properly calculate nonlinear Landau damping. For the presently considered DIII-D antenna length of $\mathrm{L}_{\mathrm{z}}=160 \mathrm{~cm}$, taking an "effective" length of $80 \mathrm{~cm}$ (since the pump wave amplitude decreases along the length of the antanna) we find that $\gamma \mathrm{L}_{\mathrm{z}} / \mathrm{V}_{\mathrm{gz}}=24$. Therefore the estimated power threshold is $\pi / 24 \times 1.25 \mathrm{MW}=0.16 \mathrm{MW}$, (the growth rate for quasi mode decay is proportional to $\mathrm{E}_{0}{ }^{2}$ ) and at 1 MW input power level we expect complete pump depletion. It should be remarked, however, that the convective losses are based on the group velocity calculated from the approximate dispersion relationship of Eq. 8 and in addition, the resonance cone behaviour of helicon waves and impact of poloidal magnetic field has been neglected in this calculation. While thresholds are easily exceeded in the present experimental situation because of the relatively low electron temperatures near the lower hybrid layer, at higher edge electron temperatures in reactor relevant plasmas the effective growth rates would be reduced and it is not clear that PDI would be important. We shall carry out more extensive calculations in the near future for such candidates for burning plasmas, such as ITER or beyond.

\section{Acknowledgements}

We wish to thank Dr. Ron Prater for providing the experimentally relevant pump wave electric fields in the early phases of this work, Dr. Charles Moeller for helpful discussions regarding the proposed antenna design, and Dr. Craig Petty for careful reading of this manuscript and helpful comments.

\section{References}

[1] R. Prater, C. P. Moeller, R. I. Pinsker, et al, Nucl. Fusion 54, 083024 (2014).

[2] R. I. Pinsker, C. P. Moeler, J. P. Anderson, et al, Paper presented at this conference.

[3] S. C. Jardin, et al, Fusin Eng. Design 38, 27 (1997).

[4] V. L. Vdovin, Plasma Physics Reports 3995 (2013).

[5] M. Porkolab, Phys. Fluids 20, 2058 (1977).

[6]. M. Porkolab, Nucl . Fusion 18, 043009 (1978).

[7] S. G. Baek. R. R Parker, P. T. Bonoli. et al, Nucl. Fusion 55, 043009 (2015).

[8] R. Cesario, A. Cardinali, C. Castaldo, et al, Nucl. Fusion 46, 462 (2006).

[9] S. J. Wang, H. H. Wi, H. J. Kim, et al, Nucl. Fusion 57, 046010 (2017)

[10] M. Porkolab and R. P. H. Chang, Rev. Modern Phys. 50, 745 (1978).

[11] M. Porkolab, Phys Fluids 17, 1432 (1974).

[12] Y. Takase and M. Porkolab, Phys. Fluids 26, 2992 (1983)

Work supported by DOE contracts DE-FC02-04ER54698 at DIII-D, a DOE FES User Facility, and Grant DE-SC001-6154 at MIT. 